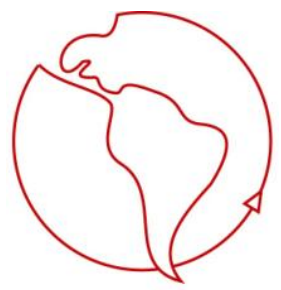

\title{
La Realidad de lo Improbable: Colaboración, una manifestación social marginal
}

Anahí Urquiza, Cynthia Meersohn y María José Torrejón

Programa de Magíster en Antropología y Desarrollo. Universidad de Chile

proyectos_antropologia@yahoo.com

\section{Resumen}

La sociedad contemporánea se observa a sí misma como una sociedad en crisis, donde el individualismo estaría debilitando los vínculos sociales. Sin embargo, podemos encontrar importantes manifestaciones de acciones colaborativas. En este artículo problematizamos esta aparente contradicción e intentamos desarrollar herramientas teóricas para observarla.

\begin{abstract}
Contemporary society observes itself as a society in crisis, where individualism is weakening the social bonds. However, we can find important facts of collaborative actions. In this paper we lay out this apparent contradiction and we intend to develop theoretical devices to observe it.
\end{abstract}

Palabras Claves: acoplamiento estructural, autodescripciones, colaboración, individualismo, solidaridad.

\section{Presentación}

Frente a las abundantes observaciones sobre el debilitamiento de los vínculos sociales en Chile, como efecto colateral de la modernización económica del país, surge la pregunta por éstos vínculos y la intención de explicar cómo funcionan en la sociedad contemporánea.

Podemos identificar una diversidad de referencias a los vínculos sociales a partir de las comunicaciones sobre cooperación, solidaridad, responsabilidad social, caridad y voluntariado, entre muchas otras. Aunque cada una de ellas refiere a una variante distinta de éstos, todas tienen una plataforma común que nosotros observamos a través del concepto colaboración social, entendido como acciones por el beneficio mutuo ${ }^{1}$.

La presente investigación pertenece a una de las temáticas del proyecto DI SOC 04/14-2 "Colaboración cultura y desarrollo" auspiciado por la Universidad de Chile y cuyo investigador responsable es el Dr. Marcelo Arnold, profesor del Departamento de Antropología y Director del Magíster en Antropología y Desarrollo de la misma institución. Este proyecto se trata de un estudio antropológico social, teórico y empírico, que trabaja en tres áreas: la construcción de un Programa de Observación de la Colaboración, la descripción de los discursos que circulan en torno al tema y la investigación empírica de prácticas colaborativas, a través de su manifestación en el trabajo voluntario.

En esta ponencia presentamos un avance del Programa de Observación. Aquí pretendemos responder a las siguientes interrogantes: ¿Cómo se observan los vínculos sociales en la sociedad contemporánea? ¿Cómo

\footnotetext{
${ }^{1}$ Este concepto proviene de colaborar, definido por la Real Academia Española como trabajar con otros en el logro de algún fin.
} 
se tematiza la colaboración social en la comunicación de expertos? ¿Cómo a partir de las teorías y métodos científicos podemos tratar las vinculaciones sociales colaborativas? A partir de esto, pretendemos construir un Programa que nos permita observar las condiciones en que la acción colaborativa se hace posible, investigando los mecanismos que subyacen a ella.

Nuestro trabajo tiene como base un enfoque sistémico-constructivista y una estrategia metodológica de observación de segundo orden ${ }^{2}$. Es desde esa opción metodológica que problematizamos las comunicaciones sobre los vínculos sociales en la sociedad contemporánea y las herramientas teóricas que nos permiten explicarlo.

\section{Descripciones de la Sociedad Contemporánea: Improbabilidad de la colaboración}

En la comunicación de la sociedad, tanto los discursos conservadores como los liberales plantean que los valores dominantes, concentrados en el individualismo y la indiferencia, acrecientan el desinterés por la responsabilidad colectiva, originando impactos negativos de todo orden. Estas descripciones se reúnen en torno al concepto de crisis, donde podemos encontrar un consenso en la observación de que la sociedad moderna se ve enfrentada a las consecuencias de su propio operar que hoy día están cuestionando las bases que le dieron forma. Una de las transformaciones identificadas como propia de este fenómeno, es el proceso de individualización, proceso que es explicado a partir del debilitamiento de las instituciones tradicionales y de los cambios en la organización de la economía mundial.

Ulrich Beck plantea que en el proceso de modernización se produce un impulso social de individualización, donde los seres humanos son desprendidos de las condiciones tradicionales de clase y familia y son remitidos a sí mismos y a su destino laboral individual. Incluso, desde esta perspectiva, se eliminan las categorías tradicionales de los grupos como clases, estamentos o capas, por lo que enfrentaríamos un capitalismo sin clases pero con todos los problemas de estructura y desigualdad social (Beck 1998). En este mismo sentido, Anthony Giddens define la modernidad como un orden postradicional, donde la razón crítica moderna atraviesa la vida social, debilitando las seguridades y hábitos de la tradición sin entregar una certidumbre que logre reemplazarla (Beck 1997:35). Alain Touraine tematiza este fenómeno a partir del concepto desintitucionalización, entendido como el debilitamiento o la desaparición de normas codificadas, lo que sumado a la desaparición de los juicios de normalidad que eran aplicados a las conductas regidas por instituciones, permite identificar la crisis de la Proto-modernidad (Touraine 1997:45).

Este proceso de individualización se refiere a un nuevo modo de socialización, a un cambio de forma en la relación entre individuo y sociedad, donde las crisis sociales son vividas como crisis individuales (Touraine 1997:45). Para Castells, este proceso se explica a partir de la individualización del trabajo, como consecuencia de la reorganización de la economía. La nueva economía se organiza en torno a redes globales de capital, gestión e información y el acceso al conocimiento tecnológico que constituye la base de la productividad y la competencia. En este escenario el trabajo es cada vez más individualizado, perdiendo su identidad colectiva, ya que al responder a la individualización de las capacidades laborales, a las condiciones laborales, y a los intereses y proyectos, se construye como una identidad individual dejando de ser un refugio social (Castells 1997 v1).

\footnotetext{
${ }^{2}$ La observación de segundo orden corresponde a una metodología constructivista que en términos generales puede ser definida como la observación de observaciones (Arnold, 1998)
} 
Desde la perspectiva de Ulrich Beck, las instituciones de la sociedad industrial, se presuponen unas a otras y al estar siendo sometidas a procesos de desvinculación y revinculación, se enfrentan a una nueva forma de conducir y organizar la vida, que no es obligatoria y no se vincula a modelos tradicionales. En este escenario, individualización significa la desintegración de las certezas de la sociedad industrial acompañada de nuevas interdependencias globales, donde la individualización y la globalización son dos caras del mismo proceso, el que no está basado en una libre decisión, por el contrario estaríamos condenados a él (Beck 1997). En este mismo sentido, Manuel Castells plantea que nuestras sociedades se estructuran cada vez más en torno a una posición bipolar entre la red y el yo, donde las redes globales de intercambios instrumentales conectan o desconectan de forma selectiva individuos, grupos, regiones o incluso países según su importancia para cumplir las metas procesadas en la red, fragmentando los movimientos sociales (Castells 1997:31-57).

Nos enfrentamos a una pérdida de fe en el progreso, que se ve reflejada en las descripciones de la sociedad contemporánea donde se comunica un malestar general frente a las consecuencias que tiene éste para los individuos y su entorno ecológico. Por ejemplo, para Anthony Giddens el mundo se ha convertido en un lugar "espantoso y peligroso", lo que nos ha obligado a dejar a un lado el sueño de que la modernidad nos conduciría a la formación de un mundo más feliz y seguro (Giddens 1993:23). Enfrentaríamos el sentimiento de que la vida no tiene valor alguno que ofrecer, lo que se traduce en una carencia de significado personal, a partir de la represión de las cuestiones morales que la vida cotidiana plantea, donde el aislamiento existencial es una separación de los recursos morales necesarios para vivir en plenitud (Giddens 1993:42).

Ahora, en lo que se refiere a las descripciones de América Latina, debemos destacar que las consecuencias no deseadas de la modernización son observadas como vividas de una forma aún más dura en nuestra Región. Esto se debería a que nuestros países son obligados a incorporarse al sistema global con visibles desventajas comparativas. Las consecuencias negativas, que fueron descritas en los párrafos anteriores, se agudizarían por la pobreza y las deficiencias institucionales para enfrentar los problemas modernos.

En este sentido, el sociólogo chileno Fernando Robles plantea que los procesos de individualización problematizados para la sociedad moderna, son vividos en Latinoamérica como individuación. Distingue entre estos dos procesos entendiendo la individualización como la configuración de la individualidad en un proceso de autoconfrontación asistido, mientras que la individuación es la configuración de la individualidad en un proceso de autoconfrontación desregulada, que se caracteriza por un aumento de las inseguridades ontológicas condicionadas por las debilidades institucionales para enfrentar los desafíos de la modernización. El proceso de individualización en los países desarrollados se viviría como un "haz de tu vida lo que quieras", mientras que en los países de América Latina la individuación se vive como un "arréglatelas como puedas" (Robles 2000).

Por otra parte, desde la perspectiva del PNUD se identifican lo que llaman "malestares culturales", que penetran la vida privada y familiar, la política, la economía, la conducta cívica y la sociedad en general (PNUD 1995). Estos malestares se basan en la sensación de inseguridad existencial y de futuro, acompañada de un escepticismo sobre las instituciones políticas y sociales. En Latinoamérica se padecería de una erosión del sentido de pertenencia, acompañado por un sentimiento de estar expuesto a formas de discriminación e intolerancia, donde el escepticismo en las instituciones políticas se expresa en la desconfianza y falta de credibilidad hacia las instituciones y prácticas democráticas. Además, existiría un "malestar ético" donde se cuestionan las normas vigentes, se expande el relativismo, se desdibujan los valores, y en general retrocede la influencia de las instituciones tradicionales, lo que provocaría una profunda crisis de sentido (PNUD 1995: 111-112). 
En definitiva, la erosión cultural que viven nuestros pueblos deja un vacío donde se conforma el escenario para el proceso de atomización de los individuos, los cuales, al experimentar su existencia de forma aislada e incomunicada, no logran descubrir su condición de sujetos activos o ciudadanos, debilitando profundamente las posibilidades de actuar colectivamente para el desarrollo de la Región.

Ahora, en lo que se refiere a la sociedad Chilena, recientes estudios nos hablan de una crisis de los vínculos sociales que constituyen su capital social y concluyen que esta situación podría constituirse en una importante barrera para su desarrollo, pues indica un debilitamiento de sus bases de confianza, sociabilidad y compromiso cívico. El incremento de las orientaciones individualistas formaría parte de esta crisis y la falta de interés en el bien común sería una de sus principales carencias. Según el Informe 1998 del PNUD, en Chile, el nivel de desconfianza interpersonal es muy alto. Sólo el 8,2 por ciento de los entrevistados (de una muestra de las grandes ciudades del país) cree que se puede confiar en las personas, la confianza se reduce y retrotrae a los contactos más intensos, se restringe a los círculos íntimos de familiares y amigos. Lo público aparece ocupado por un otro anónimo y, a veces, amenazador, del cual se tiende a esperar más una actitud agresiva que una cooperativa.

En nuestro país existiría una creciente individualización, donde las tradiciones colectivas pierden fuerza y cada chileno debe definir por su cuenta sus proyectos de vida, sus lazos y compromisos sociales. Este proceso, mientras favorece la emancipación de viejas trabas y entrega una mayor libertad para que los individuos elijan su modo de vida, debilita los referentes colectivos, provocando una excesiva individualización. Este tipo precario de identidad social puede alterar la convivencia, pues tiende a fomentar la desconfianza, el oportunismo y la desafección social. La identidad colectiva parece ser activada sólo por la fusión emocional en momentos extraordinarios, lo que no es suficiente para configurar una identidad nacional fuerte, ya que esta identidad aparece vaciada de una experiencia de sociedad. Gran parte de los chilenos no alcanzan a vivir como un sujeto colectivo, por lo débil que aparece la imagen de Nosotros (PNUD 2002: 33-36). Cuando la identidad social es débil nos sentimos rodeados de extraños en los cuales es difícil confiar e igualmente difícil proyectarnos para tomar en consideración sus intereses, así como creer que ellos tendrían esas mismas consideraciones con nosotros.

A partir de estas descripciones, hemos caracterizado a nuestra sociedad como compuesta por individuos que han perdido la ilusión de la asociatividad. Una sociedad que es percibida como algo externo y agobiante, donde se ha profundizado la exclusión social. Una sociedad donde las consecuencias no esperadas de la modernidad hacen estragos, una sociedad agresiva, individualista, desconfiada, envuelta en un deterioro de lo público.

La sociedad chilena, al igual que la sociedad global, es descrita a través de sus malestares y debilidades, donde la actitud comunitaria está en crisis. Cuando los vínculos sociales se encuentran debilitados, las acciones colaborativas, que implican trabajos por un beneficio común, son improbables. No nos podemos plantear acciones conjuntas si las realidades que van más allá de nuestros cercanos se nos presentan como ajenas.

Es por medio de estas perspectivas que se construye el imaginario de sociedad, donde las personas se reconocen y realizan como parte de ella. Y es desde estas mismas descripciones de donde surgen las demandas por una actitud social donde se fortalezca el vínculo comunitario. Se entiende que el debilitamiento de este vínculo fue uno de los precios de la instauración del llamado modelo liberal. El precio es observado como inevitable, sin embargo se demanda el rescate de este vínculo para lograr una sociedad más humana. 
Como observadores de segundo orden, podemos identificar que la sociedad contemporánea se enfrenta a sus propias descripciones, las que coinciden en una descripción negativa de los valores dominantes (Arnold 2004:1). Son descripciones de denuncia, que pretenden explicar los problemas de nuestra sociedad responsabilizando a elementos aislados como el individualismo, la crisis institucional, el neoliberalismo, etc. Describen una sociedad llena de malestares y desconfianzas, una sociedad que perdió los referentes que apuntaban a una actitud comunitaria. Ahora, si aceptáramos estás descripciones como "reales", tendríamos que observar la colaboración como algo aislado y absolutamente improbable.

\section{Colaboración y la viabilidad de lo social}

Cuando confrontamos estos discursos con las explicaciones científicas sobre la construcción de lo social, nos enfrentamos a un grave problema: la colaboración está en la base de la viabilidad de lo social.

Las sociedades humanas han sido investigadas desde diversas disciplinas y perspectivas teóricas. Una de las preocupaciones fundamentales ha sido comprender la vida del ser humano en comunidad. Somos un animal gregario y desde esta perspectiva se han dado algunas respuestas. Desde lo biológico y lo social, se presenta a la colaboración como un pilar de nuestra vida en sociedad. En lo que sigue presentamos dos importantes posturas antropológicas al respecto, la de Ashley Montagu y Marcel Mauss, respectivamente.

Ashley Montagu, intenta comprender, desde sus fundamentos biológicos, el comportamiento cooperativo entre los seres humanos. Plantea, que el principio dominante de la vida social, no es la lucha por la existencia en la competencia, sino que la cooperación, entendiéndola como el comportamiento interactivo entre organismos que se benefician mutuamente para su supervivencia (Montagu 1969:36). No hay seres vivos que existan solos, incluso los organismos más inferiores presentan tendencias innatas hacia algún tipo de vida social, comportamiento que puede limitarse a la breve asociación sexual, al periodo de incubación o llegar a formar complejas comunidades sociales, como las humanas (Montagu 1969:36-37). Entonces, todo ser vivo, en algún momento de su existencia, vive en algún tipo de comunidad, y para esto es necesario cierto tipo de cooperación.

El comportamiento cooperativo en organismos vivos se entiende gracias a la existencia de un impulso para formar agregados sociales. Montagu llama a este impulso socialidad, el que sería una cualidad presente en todos los organismos vivos, pero que es más fuerte en algunos organismos que en otros. Entonces, todos los seres vivos tienen una naturaleza social que encuentra su origen en la dependencia que se establece en la relación reproductora entre organismo padre y vástago (Montagu 1969:36). En definitiva, la tendencia a formar sociedades surge con la vida misma y la sociedad humana sería la culminación de esta tendencia. Esto no pretende rebatir que la homeostasis, entendida como principio de autoconservación, es el impulso dominante del organismo, pero es en la realización de este impulso donde el organismo necesariamente debe cooperar con otros organismos. Llegando aun más lejos, Montagu explica que el perfeccionamiento de las especies se logra a partir de estos procesos de cooperación, ya que es un factor de estabilización que contribuye al éxito del grupo. Esto se entiende, porque la probabilidad de la supervivencia del grupo aumenta con el grado en que se ajustan los individuos entre ellos y con su medio (Montagu 1969).

Entonces, desde la perspectiva de este autor, la cooperación se constituye en una condición de la existencia de los seres vivos. En el caso de los seres humanos la cooperación ha sido fundamental, ya que al ser un animal proveído de pocas herramientas corporales para enfrentar la naturaleza se ha visto obligado a vivir siempre en cooperación con otros individuos de su misma especie para sobrevivir. De esta forma, la sociedad humana sólo ha sido posible a partir de las acciones cooperativas. 
Por otra parte, Marcel Mauss (1971) indaga en las profundidades de la constitución de lo social a partir de la investigación que realiza sobre el Sistema de Prestaciones Totales. Aquí identifica a la reciprocidad como uno de los grandes pilares de las sociedades humanas.

El potlach es identificado como un proceso donde se constituye el vínculo social. A partir de la destrucción festiva de los objetos, surgen vínculos que establecen un principio de comunidad más allá de las cosas y de su utilidad, donde devolver el don recibido es el fundamento de este vínculo, es en este acto donde está el carácter integrativo del Sistema de Prestaciones Totales. Este sistema funciona a partir de la circulación obligatoria de riquezas, tributos y dones, donde ofrecer una cosa a alguien es ofrecer algo propio, hay que entregar algo al otro que es parte de nosotros, y asimismo, aceptar algo de alguien es aceptar parte de su esencia espiritual. De esta forma, se crea comunión y alianza a partir del intercambio de dones (Mauss 1971).

Las prestaciones estudiadas por Mauss, tienen un carácter voluntario, aparentemente libre y gratuito, pero finalmente, obligatorio e interesado. Son hechos sociales totales o generales, es el movimiento de un todo, basado en la reciprocidad donde se beneficia tanto el individuo como el grupo.

En definitiva, hemos identificado cómo la evolución social del hombre y sus perspectivas de desarrollo futuro están estrechamente ligados con una acción conjunta para el beneficio mutuo. Al parecer, la sociedad humana se ha constituido en lo que es, a partir de este tipo de acciones colaborativas. Entonces, la colaboración no ha sido una forma marginal de organización social, como parece serlo en la actualidad, por el contrario, ha sido uno de los pilares de nuestro desarrollo como humanidad.

Por otra parte, además de ser un elemento fundante de lo social, la colaboración se presenta como un elemento imprescindible para la convivencia humana y para el desarrollo económico de nuestras sociedades. La perspectiva teórica que se centra en las Redes Sociales y el Capital Social, nos dan cuenta de esta postura. En este enfoque teórico, se observan las redes sociales, como las estructuras de nuestras sociedades, las que están construidas a partir de actores sociales que establecen vínculos entre ellos. Cuando estos se transforman en relaciones recurrentes de reciprocidad, son identificados como capital para los individuos y para la comunidad.

El estudio de Redes Sociales ha sido importante en las Ciencias Sociales desde sus inicios. Podemos encontrar sus antecedentes en autores clásicos como Durkheim, Marx, Simmel, Nadel y Barnes, donde las redes de relaciones sociales son parte importante del objeto de la sociología. Hoy día ya se han consolidado los temas abordados por el análisis de redes sociales, como el estudio de la red Internet, redes de organizaciones, redes de acción política, redes personales, redes de ayuda mutua, etc. Pero sin duda, una de las áreas más trabajadas es la que tematiza estás redes sociales a partir del concepto de capital social (Kliksberg 2000).

Una red social es un conjunto de actores entre los que se establecen una serie de vínculos. En el análisis de una red se considera la estructura de las relaciones en las que cada actor se encuentra involucrado, incluso, estos actores son descritos a partir de sus vínculos sociales. Aquí las estructuras que se buscan, son regularidades en las formas de vinculación que emergen en los conjuntos relacionales. Este tipo de análisis, presupone que las características estructurales de las redes de relaciones sociales determinan los comportamientos de los individuos implicados en ellas. 
Pero, ¿cuál es la importancia de observar estos vínculos? Desde diversas perspectivas de análisis se ha identificado a los vínculos sociales como fuente de bienestar emocional, material y social. Las redes sociales son utilizadas como medio de integración y de implicación de las personas en función de un sistema social determinado (Requena Felix 1994). Esto ha llevado a que uno de los principales campos de aplicación de la perspectiva de redes sociales sea el apoyo social, entendido como los recursos sociales con los que cuentan los individuos para su bienestar. Es en este campo de aplicación que nos enfrentamos al concepto de "capital social", el que indica las cualidades de la organización social que se transforman en activos para las personas que participan en ella. Esto se refiere a características de la organización social, como redes, normas, y confianza social, que facilitan la coordinación y la cooperación para la ventaja mutua (Putman 1994).

Entonces, desde esta perspectiva se entiende que las redes sociales de un actor social (persona o grupo) constituyen un activo de suma importancia, al que puede recurrir en momentos de crisis, disfrutar como un fin en si mismo y, también, utilizar para obtener ganancias materiales. En efecto, se identifica que aquellas comunidades que cuentan con un abanico diverso de redes sociales y asociaciones cívicas, se encuentran en mucho mejor pie para enfrentar la pobreza y la vulnerabilidad, para solucionar conflictos y aprovechar nuevas oportunidades. Podemos identificar un consenso respecto de la importancia de las relaciones sociales (conceptualizadas como capital social) en el tema del desarrollo, donde éstas constituyen una oportunidad de movilizar recursos que aumentan el crecimiento de una sociedad, cuando las redes sociales están basadas en principios de confianza, reciprocidad y normas de acción, que facilitan la acción colectiva (Bullen y Onyx 1998).

Como podemos ver, la colaboración se presenta como un elemento imprescindible para la constitución de lo social, para su mantenimiento y para el desarrollo de nuestras sociedades. Entonces, enfrentando las tendencias identificadas en las descripciones de la sociedad contemporánea, nos vemos obligados a preguntarnos: ¿Nuestra sociedad se está enfrentando a sus propios límites? ¿Estamos poniendo en peligro la viabilidad de lo social? Porque si efectivamente nuestra capacidad para vivir en comunidad se ha deteriorado, ¿nos enfrentamos a tendencias autodestructivas? o, ¿debemos cuestionar éstas observaciones?

\section{Colaboración: ¿una manifestación marginal?}

En primer lugar expusimos cómo en las descripciones de la sociedad contemporánea, las acciones colaborativas son identificadas como improbables. Hemos observado además, que desde importantes perspectivas teóricas se identifican las acciones por el beneficio mutuo, acciones colaborativas, como fundamentales para la constitución de lo social, lo que nos llevó a preguntarnos si estamos en el límite de la viabilidad social. Ahora agregaremos un nuevo componente a la discusión.

De forma paralela a estas autodescripciones (Luhmann 1997), podemos identificar importantes comunicaciones en torno acciones colaborativas. Esto lo observamos en algunas de sus expresiones más clásicas como son las vinculadas a lo que hoy día llamamos solidaridad. Ahora nos proponemos mostrar algunos datos sobre este tipo de acción colaborativa.

Las manifestaciones de acciones solidarias han sido descritas principalmente a través del voluntariado. Existen antecedentes de importantes movimientos en torno a este tipo de acciones a nivel mundial. Algunas cifras que nos pueden ilustrar esto han sido recogidas de la Encuesta Mundial de Valores ${ }^{3}$ (1990

\footnotetext{
${ }^{3}$ Encuesta anual realizada por el Worldwatch Institute que investiga los cambios socioculturales y políticos en más de setenta países, abarcando cerca del $80 \%$ de la población mundial.
} 
y 2000). Estas cifras indican que en diversos países los voluntarios constituyen un porcentaje significativo de la fuerza de trabajo total. En países como los nórdicos, algunos de Europa Occidental, Canadá, Estados Unidos e Israel, el voluntariado es un aporte muy significativo al Producto Bruto Nacional. Por ejemplo, en Israel un $25 \%$ de la población realiza tareas voluntarias de modo regular y genera bienes y servicios equivalentes al 8\% del PIB. En un país tan "individualista" como Estados Unidos el aporte es del 5,6\%, con un volumen de voluntariado cercano al 30\% (85 millones) de la población total del país. Otros países con cifras importantes en este sentido son: España (0,6\% del PIB, $11 \%$ volumen de voluntariado), Argentina (0,9\% del PIB, $15 \%$ volumen de voluntariado), y Brasil (1,2\% del PIB, 11,5\% volumen de voluntariado).

Ahora, respecto a lo que sucede en Chile, en la Investigación realizada por FLACSO CHILE-MORICERC en el año 2002, se indica que de cada 100 personas 28 son voluntarios. Con una participación en diferentes grados de compromiso: un 66\% declara haber tenido alguna relación con organizaciones de este tipo, un $44 \%$ haber asistido a reuniones, un $36 \%$ haber donado dinero, un $20 \%$ declara ser miembro de alguna de ellas, $17 \%$ realizar trabajo voluntario, un $42 \%$ declaran haber participado "una vez en la vida" en alguna organización y un $19 \%$ declara haberlo realizado en los últimos 12 meses. Es interesante destacar que $36,4 \%$ de estos voluntarios dedica entre dos y cinco horas a la semana a esta labor y un $22,5 \%$ le dedica entre cinco y diez horas semanales. El promedio de horas de dedicación al trabajo voluntario es de 10,8 horas semanales (FLACSO CHILE-MORI-CERC, 2002).

Al observar los datos entregados por los estudios presentados, nos puede parecer que contradicen las tendencias identificadas en las autodescripciones de la sociedad contemporánea. Sin embargo, también se pueden observar ambas tendencias como parte de un mismo proceso, ya que mientras se comunique sobre ellas coexisten, son simultáneas. Pero frente a esto, ¿cómo lo explicamos?, ¿cómo es que se dan ambas tendencias? Para enfrentar este problema debemos entender a qué responden, construyendo y aplicando herramientas teóricas que nos permitan explicar esta coexistencia de lógicas aparentemente excluyentes.

\section{Herramientas Teóricas}

Podemos identificar dos grandes herramientas teóricas para explicar la coexistencia de las tendencias individualistas con acciones colaborativas. Estas herramientas son: la Teoría de la Elección Racional y la Teoría de la Autopoiesis de los biólogos chilenos Humberto Maturana y Francisco Varela.

En la Teoría de la Elección Racional podemos encontrar aportes importantes para observar la colaboración como comportamiento individual. En esta teoría, se intenta abarcar tanto los aspectos individuales como las propiedades estructurales, pretendiendo explicar como actúa un sujeto en una situación social determinada. Ahora, su supuesto central es que los individuos toman sus decisiones en función de cuáles son los resultados "anticipados" de sus acciones y eligen las acciones que creen que acarrearan algún tipo de mejora en sus vidas (Mari-Klose 2000:55-85)

Para entender el fenómeno de la colaboración, observado desde esta perspectiva, utilizamos el concepto acción colectiva ${ }^{4}$. Los individuos eligen entre cooperar y no cooperar y la acción colectiva intenta establecer las condiciones en que la cooperación se produce, tratando de explicar la existencia y acción de un colectivo, es decir, cuándo y cómo cierto número de personas actuarán conjuntamente con un mismo propósito. Aquí se plantea que las personas se muestran reacias a salir de la inactividad, a pesar de que la

\footnotetext{
${ }^{4}$ Es a partir del concepto acción colectiva que en la Teoría de la Elección Racional se ha problematizado la cooperación entre individuos que persiguen un mismo fin.
} 
mejor opción colectiva sea cooperar. Esto se debe a que los individuos suelen suponer que su esfuerzo individual será superior al beneficio que podrán obtener de la acción colectiva. En este sentido, este tipo de acciones se presentan como altamente improbables. Sin embargo, a partir de la Teoría de los Juegos (Mari-Klose op.cit.) podemos observar cómo los individuos cooperan en variadas situaciones de interdependencia, donde la decisión de cooperar o no cooperar se entiende como una decisión estratégica y las ganancias o beneficios de cada agente no sólo dependen de su actuación sino también de las decisiones tomadas por el resto de agentes con los que interactúan (Mari-Klose 2000:55-61). Entonces, un individuo decide cooperar cuando considera que al hacerlo va a obtener algún beneficio individual (incentivo selectivo) o cuando evalúa que su participación va a tener los efectos esperados en la situación de interdependencia en la que se encuentra (Mari-Klose 2000:28-29).

Por otra parte, en la propuesta de Maturana y Varela, la teoría de la autopoiesis, se explica la organización del ser vivo como un operar cerrado de producción de componentes producidos por la misma red de relaciones de componentes que los generan, esto es lo que ellos llama autopoiesis, que significa autoproducción. Entonces, todo organismo organiza su propia estructura y produce internamente sus componentes. Estos sistemas se encuentran clausurados operacionalmente, pero esto no implica una ausencia de interacciones entre el sistema y su medio. Por el contrario, ningún sistema opera en la nada, requiere condiciones previas de las cuales depende su existencia. Estos sistemas utilizan materiales y energía que no pueden autogarantizarse, donde su disponibilidad es parte de las condiciones de existencia del sistema. Sin embargo, estas condiciones no determinan el curso de las operaciones internas del sistema, solamente las posibilitan.

Ahora, a través del concepto acoplamiento estructural se observan las interacciones recurrentes entre un ser vivo y su entorno, indicando las interacciones entre sistemas autopoiéticos que mantienen una deriva co-ontogénica donde se gatillan mutuamente cambios estructurales. En estas interacciones recurrentes emerge un dominio común de coordinaciones, a partir de los cuales se construyen unidades de órdenes mayores. Del acoplamiento entre sistemas celulares emergen unidades autopoiéticas de segundo orden que descansan en la autopoiesis de las de primer orden, pero desarrollan su propia organización permitiéndoles operar clausuradamente. Desde esta perspectiva, lo social es resultado de interacciones humanas, que a través del lenguaje, interactuaron recurrentemente entre sí logrando dar lugar a acoplamientos de tercer orden, que es lo que nosotros llamamos sistemas sociales.

Desde esta perspectiva, el lenguaje ha sido central para modificar y ampliar los dominios conductuales de los seres humanos. Incluso es la aparición de la interacción mediada por el lenguaje, lo que da origen al modo de vida humano. Desde esta interacción surge la observación humana, la conciencia y la reflexión, ya que permite traspasar los límites de los organismos individuales hasta alcanzar el dominio de la convivencia social.

Maturana llega aún más allá en su explicación y entiende la sociabilidad humana como un modo de vida basado en la cooperación, como coordinación conductual aprendida. Visualiza la importancia de la convivencia entre los individuos y trabaja el concepto Amor para entender las conductas en las que tratamos al otro como legítimo otro en convivencia con nosotros. Lo importante de estas conductas es que al aceptar la legitimidad del otro nos hacemos responsables de nuestra relación con él, por esto el amor es la emoción que funda lo social (Maturana 1991:36).

En definitiva, desde esta perspectiva se entiende que la conducta de un individuo que se refiera a la conservación del grupo, también es la expresión de su acoplamiento estructural en un medio que incluye al grupo y por lo tanto es expresión de su conservación, de su adaptación como individuo. No hay 
contradicción por lo tanto en la conducta del individuo mientras realiza su individualidad como miembro del grupo: "es altruistamente egoísta y egoístamente altruista porque su realización individual incluye su pertenencia al grupo que integra" (Maturana y Varela 1984:131).

A partir de estas herramientas teóricas, podemos comprender la coexistencia de dos tendencias aparentemente excluyentes, el individualismo y las acciones colaborativas, como parte de un mismo proceso, donde los individuos que colaboran con otros están velando por su propia existencia: "Todo ocurre como si hubiese un balance entre la mantención y subsistencia individual y la mantención y subsistencia del grupo como unidad más amplia que engloba al individuo. De hecho, en la deriva natural se logra un balance entre lo individual y lo colectivo en la medida en que los organismos al acoplarse estructuralmente en unidades de orden superior (que tienen su propio dominio de existencia), incluyen la mantención de éstas en la dinámica de su propia mantención” (Maturana y Varela 1984:131).

Cuando nos referimos a las acciones colaborativas, podemos hablar de un Altruismo Egoísta, donde el individuo actúa a partir de incentivos selectivos que lo motivan a colaborar con el otro, cuando evalúa que su acción obtendrá los beneficios esperados. Esto se entiende, porque cuando el individuo actúa por el grupo vela por su propia sobrevivencia a través de la viabilidad del colectivo.

\section{Conclusiones Preliminares}

Aún cuando los discursos sobre nuestra sociedad, como las descripciones de la modernidad, y las explicaciones de los procesos que guían las decisiones cotidianas, apuntan a que el fenómeno de la colaboración es altamente improbable, podemos observar innumerables expresiones de este tipo. Además, importantes teorías científicas indican que la colaboración es la base de la sociabilidad humana.

Desde nuestra perspectiva, la colaboración social está subestimada por una modernidad que se observa a través del individualismo y la debilidad de la identidad colectiva, relegando la acción por el beneficio común (colaboración) a un papel secundario. Las descripciones de la sociedad no sólo plantean como improbable las acciones de este tipo, además no logran explicar su presencia. Existe una predominancia de las explicaciones basadas en la lógica competitiva que tiende a invisibilizar la colaboración, observando estas acciones como fenómenos residuales. Así mismo, estas descripciones podrían inhibir las acciones colaborativas ya que pueden influir en la evaluación que hace el individuo sobre los beneficios esperados de su acción.

Nosotros podemos decir que caminamos hacia la construcción de un Programa de Observación para las acciones colaborativas, que intentará romper con esta dinámica de invisibilización. Nuestra pretensión es que el concepto colaboración se constituya en una distinción que nos permita observar un fenómeno global, donde expresiones aparentemente excluyentes, como el individualismo y la solidaridad, puedan ser explicadas. Para esto, basándonos en el Programa Sociopoietico ${ }^{5}$, observamos las comunicaciones colaborativas como distinciones que se utilizan para problematizar las acciones que se orientan al beneficio individual, pero considerando al beneficio del entorno como parte de este beneficio.

\section{Bibliografía}

\footnotetext{
${ }^{5}$ Programa de Observación trabajado por el Dr. Marcelo Arnold, donde se proponen herramientas para observar la policontextualidad de la sociedad contemporánea a través de la observación de segundo orden.
} 
Arnold, Marcelo. 1998. Recursos para la investigación sistémico/constructivista. Cinta de Moebio No.3. Facultad de Ciencias Sociales. Universidad de Chile.

Arnold, Marcelo. 2004. Sociopoiesis: Fundamentos de la Observación de Segundo Orden. MS.

Beck, Ulrico. 1998. La sociedad del riesgo. Hacia una nueva modernidad. Ed. Paidos, Barcelona.

Beck, Ulrich et. al. 1997. Modernización reflexiva. Política, tradición y estética en el orden social moderno. Alianza Universidad, Madrid.

Bullen, Paul y Jenny Onyx. 1998. Measuring social capital in five communities in NSW. Center for Australian Community Organizations and Management, Working Paper Series 41, Sydney.

Castells, Manuel. 1997. La era de la información. Economía, sociedad y cultura. Ed. Alianza, Madrid

FLACSO CHILE-MORI-CERC. 2002. Investigación sobre la conversación social y opinión pública acerca del voluntariado en Chile. DOS-SEGEGOB. Santiago

Giddens, Anthony. 1993. Consecuencias de la modernidad. Editorial alianza, Madrid.

Kliksberg, Bernardo. 2000. Capital social y cultura. Claves olvidadas del desarrollo. Banco Interamericano de Desarrollo. Disponible en http://www.iadb.org/intal/publicaciones/kliksberg.pdf

Luhmann, Niklas. 1997. Observaciones de la Modernidad. Racionalidad y contingencia en la sociedad moderna. Ed. Paidós, Barcelona

Maturana, Humberto. 1991. El sentido de lo Humano. Colección Hachette, Santiago.

Maturana, H. y F. Varela. 1984. El árbol del conocimiento. Editorial Universitaria. Santiago

Mauss, Marcel. 1971. Ensayo sobre los Dones: Razón y Forma del Cambio en las Sociedades Primitivas. Paris.

Montagu, Ashley. 1969. La Dirección del Desarrollo Humano. Editorial Tecnos, Madrid.

Pau Mari-Klose. 2000. Elección Racional. Cuadernos Metodológicos. Centro de Investigaciones Sociológicas, Madrid.

PNUD. 1995. Gobernabilidad y Desarrollo Democrático en América Latina y el Caribe. Disponible en http://desarrollohumano.cl/informes

PNUD. 1998. Las Paradojas De La Modernización. Disponible en http://desarrollohumano.cl/informes

PNUD. 2002. Nosotros Los Chilenos Un Desafio Cultural. Disponible en http://desarrollohumano.cl/informes

Putnam, Robert. 1994. Para hacer que la democracia funcione. Editorial Galac, Venezuela. 
Requena, Felix. 1994. Amigos y redes sociales. Elementos para una sociología de la amistad. Centro de investigaciones sociológicas. Madrid

Robles, Fernando. 2000. El desaliento inesperado de la modernidad. Molestias, irritaciones y frutos amargos de la sociedad del riesgo. Ediciones Sociedad Hoy, Dirección de investigación. Universidad de Concepción.

Touraine, Alain. 1997. ¿Podremos vivir juntos? Iguales y Diferentes. Fondo de Cultura Económica de Argentina. Buenos Aires.

Worldwatch Institute. 2000. Encuesta Mundial de Valores. Disponible en http://www.worldwatch.org/ 